\title{
Impact of Ileal Transposition Surgical Intervention on Antioxidant Status Measured in Liver Tissue of Obese Zucker Rats (Crl:ZUC-Lepr ${ }^{\text {fa }}$ )
}

\author{
Bronisława Skrzep-Poloczek (D), ${ }^{1}$ Dominika Stygar $\left(\mathbb{D},{ }^{1}\right.$ Tomasz Sawczyn, ${ }^{1}$ Ewa Romuk (D), ${ }^{2}$ \\ Elżbieta Chełmecka, ${ }^{3}$ Jakub Poloczek, ${ }^{4}$ Agnieszka Dulska, ${ }^{1}$ Wojciech Kazura, ${ }^{1}$ \\ and Konrad W. Karcz ${ }^{5}$ \\ ${ }^{1}$ Department of Physiology, School of Medicine with the Division of Dentistry in Zabrze, Medical University of Silesia, \\ Katowice, Poland \\ ${ }^{2}$ Department of Biochemistry, School of Medicine with the Division of Dentistry in Zabrze, Medical University of Silesia, \\ Katowice, Poland \\ ${ }^{3}$ Department of Statistics, Department of Instrumental Analysis, School of Pharmacy with the Division of Laboratory Medicine \\ in Sosnowiec, Medical University of Silesia, Katowice, Poland \\ ${ }^{4}$ Department of Rehabilitation, 3rd Specialist Hospital in Rybnik, Poland \\ ${ }^{5}$ Clinic of General, Visceral, Transplantation and Vascular Surgery, Hospital of the Ludwig Maximilian University, \\ Munich, Germany
}

Correspondence should be addressed to Dominika Stygar; dstygar@gmail.com

Received 22 July 2018; Accepted 10 October 2018; Published 11 November 2018

Academic Editor: Francisco J. Romero

Copyright (c) 2018 Bronisława Skrzep-Poloczek et al. This is an open access article distributed under the Creative Commons Attribution License, which permits unrestricted use, distribution, and reproduction in any medium, provided the original work is properly cited.

Background. The main factor characteristic for low-grade systemic inflammation typical for obesity is oxidative stress (OS). Reactive oxygen species (ROS) production is higher and more increased in time in the obese patients than in lean subjects. Aims. To assess the effect of ileal transposition (IT) and sham types of bariatric procedures on the antioxidative systems in the liver tissue of Zucker rats (Crl:ZUC Lepr $\left.{ }^{\text {fa }}\right)$. Method. 21 animals were divided into the experimental groups: control group $(n=7)$, sham group $(n=7)$, and IT group $(n=7)$. Sham and IT animals underwent selected surgery. The concentration of total antioxidant capacity (TAC), total antioxidant status (TOS), and activity of glutathione reductase (glutathione-disulfide reductase, GR, GSR), catalase (CAT), glutathione peroxidase (GPx), glutathione S-transferase (GST), and total superoxide dismutase activity (SOD) were assessed in liver tissue 3 months after surgery. Results. IT procedure significantly increased TAC when compared to sham and the control group. Animals after IT showed higher levels of TOS when compared to sham procedure. The total amount of TOS was similar in IT and control groups. GPx activity was increased in the groups submitted to the sham and IT surgery in relation to control. GR and CAT activities were significantly higher after IT in comparison to control and sham procedures. Total SOD and MnSOD were significantly higher in sham-operated animals in comparison to IT intervention and control groups. Conclusions. IT procedure had a positive impact on the diminishing of oxidative stress measured by TAC and TOS markers. The dynamic, adaptive, and protective mechanisms of enzymatic antioxidant systems were observed after the IT but not sham procedure. Nevertheless, 3 months after surgery, the midterm effect of bariatric surgery was observed, which might not fully balance the antioxidative response. 


\section{Introduction}

One of the main factors characteristic for low-grade systemic inflammation and an immune activation characteristic for obesity is OS. ROS generation and inflammation increase more in time in obese patients than in lean subjects [1].

Bariatric surgery is, so far, the most efficient procedure for achieving significant and long-term body mass reduction in morbidly obese subjects [2]. It has been also showed to induce remission and significant improvement in type 2 diabetes mellitus and fatty liver disease. Bariatric surgery lowered mortality from cardiovascular risk and improved blood pressure, dyslipidemia, and dysglycemia [2-5]. Also, Roux-en-Y gastric bypass procedure (RYGB) was reported to significantly decrease the level of lipid peroxidation [4]. Ileal transposition (IT) is a type of jejunoileal bypass, notably reducing food intake [6]. Pathophysiologically, the remission of type 2 diabetes is associated with the active delivery of aliment to the terminal ileum [7]. Nevertheless, the comparative studies on OS after IT and sham procedures were not performed. In this work, we decided to use diabetic and obese Zucker rats (Crl:ZUC-Lepr $\left.{ }^{\text {fa }}, \mathrm{ZF}\right)$ as an animal model of obesity, phenotypically close to human obesity [8].

The antioxidant defence under the conditions of OS caused by obesity considers the cumulative action of enzymatic and nonenzymatic antioxidant systems present in liver tissue [9]. The dynamic interaction between antioxidants as an answer to the IT and sham bariatric procedures is, therefore, the main aim of this study, hence giving a discernment into the sensitive balance between oxidative stress and antioxidant parameters 3 months after the surgery. This work is a new attempt at indicating the importance of comprehending the complex processes of the homeostatic control of antioxidants in liver tissue and its dynamic changes under oxidative stress development after IT and sham surgery.

\section{Materials and Methods}

2.1. Animals, Diet, and Study Design. The study was conducted according to the Guide for the Care and Use of Laboratory Animals (Directive 2010/63/EU) and the Ethics Committee of the Medical University of Silesia in Katowice, Poland. 21 male, obese12-week-old Zucker rats (Crl:ZUC Lepr $^{\mathrm{fa}}$, Charles River Breeding Laboratories, Wilmington, Mass, USA). The animals were kept under controlled conditions on a 12/12-hour light and dark cycle, in humidity $70 \pm 1 \%$, with unlimited access to water and rat food (Provimi Kliba AG, Kaiseraugst, Switzerland). Food contained 24\% of protein, $4.9 \%$ of fat, $7 \%$ of crude ashes, $4.7 \%$ of crude fibre, lysine $(13.6 \mathrm{~g} / \mathrm{kg})$, calcium $(12 \mathrm{~g} / \mathrm{kg})$, methionine $(4.5 \mathrm{~g} / \mathrm{kg})$, and phosphorus $(8.3 \mathrm{~g} / \mathrm{kg})$.

After one week of acclimatisation, the animals were assigned to three experimental groups: control group CD $(n=7)$, sham group $(n=7)$, and IT group $(n=7)$. The sham and IT groups underwent two different types of surgery: sham, which is a control type of surgery, and IT (Figure 1). The control group was not included in any type of surgical intervention.

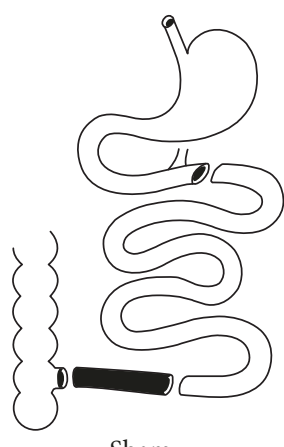

Sham

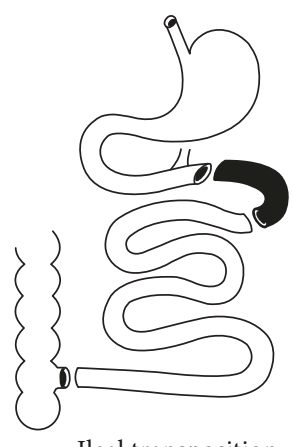

Ileal transposition
Figure 1: The scheme of the IT surgery.

2.2. Ileal Transposition and Sham Surgery. The surgery procedures were previously described by Grüeneberger et al. [7]. Isoflurane $2 \%$ with oxygen flow at $21 / \mathrm{min}$ under spontaneous breathing was used to induce and maintain anaesthesia. After an abdominal midline incision, length $4-5 \mathrm{~cm}$ was performed and the Bauhin's valve was determined. $50 \%$ of the distal ileum was localized and transected. The ileal continuity was restored by an end-to-end extramucosal anastomosis using PDS 6/0 (Ethicon, Blue Ash, OH), excluding the transposed segment. Then, the ligament of Treitz was determined, and the jejunum was divided $5 \mathrm{~cm}$ aborally. The transposed segment of ileum was inserted in an isoperistaltic fashion, and two end-to-end anastomoses were performed. For control and sham surgery, transections were performed at all three analogous points. Anastomoses were completed correspondingly, nevertheless without IT (Figure 1). Fascia and skin closures were performed as a continuous suture using Monocryl 4/0 and Vicryl 4/0. After the surgery, all rats were kept on a liquid diet for $24 \mathrm{~h}$ (Nutrison, Nutricia, Poland).

2.3. Tissue Collection. Isoflurane $2 \%$ with oxygen flow at $21 /$ min under spontaneous breathing was used to induce and maintain anaesthesia. At the end of the 12th week after surgery, corresponding to the 15th week of the experiment, the tissue of the liver was collected and the rats were euthanized. The muscle tissue $(100 \mathrm{mg}$ ) was homogenized in $1 \mathrm{ml}$ of a homogenising buffer. All samples were snap frozen in liquid nitrogen and stored at $-80^{\circ} \mathrm{C}$ until further analysis. All experimental procedures were approved by the Ethical Committee for Animal Experimentation of the Medical University of Silesia (58/2014).

2.4. Oxidative Stress Marker Analysis. The total antioxidant capacity (TAC), total antioxidant status (TOS), and the activity of the following antioxidant enzymes: glutathione peroxidase $(\mathrm{GPx})$, glutathione reductase (glutathione-disulfide reductase, GR, GSR), catalase (CAT), glutathione Stransferase (GST), total superoxide dismutase activity (SOD) in the liver tissue of the control, IT and Sham operated rats were determined. 
2.5. Total Antioxidant Capacity (TAC). TAC was measured using a commercial kit (Randox Co., England). The 2.2' azino-di-(3-ethylbenzothiazoline sulphonate) (ABTS) was incubated with a peroxidase (metmyoglobin) and hydrogen peroxide to produce the radical cation ABTS+, which has a relatively stable blue-green colour and was measured at $600 \mathrm{~nm}$. The suppression of the colour was compared to the standard for TAC measurement assays (Trolox). The assay results are expressed as a Trolox equivalent ( $\mathrm{mmol} / \mathrm{l})$. The inter- and intra-assay coefficients of variations $(\mathrm{CV})$ were $1.1 \%$ and $3.8 \%$, respectively.

2.6. Total Oxidative Status (TOS). The method according to Erel [9] uses the oxidation of iron (II) ions to iron (III) ions in an acidic medium. Then iron (III) ions with xylene orange form a colourful complex ranging up to a blue-purple colouration. Absorption readings were taken with a $560 \mathrm{~nm}$ filter on the VICTOR-X3 from PerkinElmer. The TOS level was calculated from the calibration curve using $\mathrm{H}_{2} \mathrm{O}_{2}$ as the standard. Values are expressed in $\mu \mathrm{mol} / \mathrm{l}$.

\subsection{Oxidative Enzyme Analysis}

2.7.1. Glutathione Reductase Activity (EC 1.8.1.7). GR enzymatic activity in the liver tissue was evaluated by a decrease in the concentration of NADPH in the samples using a GR buffer (200 mM sodium phosphate $\mathrm{pH} 7.5,6.3 \mathrm{~mm}$ EDTA) and kinetic reading was performed at a wavelength of $340 \mathrm{~nm}$ for 10 minutes [10].

2.7.2. Catalase Activity (EC 1.11.1.6). The catalase activity in the liver tissue was measured using Aebi methods. Briefly, a $50 \mathrm{mM}$ TRIS/HCl buffer, $\mathrm{pH} 7.4$, and perhydrol were mixed with $50 \mu \mathrm{l}$ of tissue. After 10 seconds, the absorbance was read at $\lambda=240 \mathrm{~nm}$, every 30 seconds for 2 minutes. The enzymatic activity was expressed in IU/mg protein [11].

2.7.3. Glutathione Peroxidase Activity (EC 1.11.1.9). To measure the activity of glutathione peroxidase, $40 \mathrm{mM}$ sodium azide, GSH (diluted in 5\% metaphosphoric acid), GR (GPx diluted in the buffer), NADPH (diluted with sodium bicarbonate $5 \%$ ), and $0.5 \mathrm{mM}$ tert-butyl were incubated in the liver tissue with a GPx buffer $(100 \mathrm{mM}$ potassium phosphate with $1 \mathrm{mM}$ EDTA pH 7.7). The decay of NADPH concentration was evaluated for 10 minutes in a spectrophotometer, at $340 \mathrm{~nm}[12]$.

2.7.4. Glutathione S-Transferase Activity (EC 2.5.1.18). Transferase activity of glutathione S-transferase in the liver tissue was estimated by the kinetic method, previously described by Habig and Jakoby [13]. 1-Chloro-2,3-dinitrobenzene was used as a substrate and results are expressed in $\mathrm{IU} / g$ protein.

2.7.5. Superoxide Dismutase Analysis (EC 1.15.1.1). SOD isoenzymes' activity was determined with the use of the spectrophotometric method by Oyanagui [14]. KCN was used as the inhibitor of the CuZnSOD isoenzyme. CuZnSOD activity was calculated as the difference between total SOD activity and MnSOD activity. SOD activity was calculated against a blank probe (containing bidistilled water). Enzyme activity was expressed as nitrite units (NU) per mg of protein in tissue. One NU exhibits $50 \%$ inhibition of formation of nitrite ion under the method's condition [14].

2.7.6. Protein Concentration. Protein concentration was determined by Lowry methods using bovine serum albumin as the standard [15].

\section{Statistical Analysis}

Statistical analysis was completed using STATISTICA 12.5 PL (StatSoft, Cracow, Poland.). A $p$ value below 0.05 was accepted as statistically significant. All tests were two tailed. Interval data were expressed as a mean value \pm standard deviation in the case of a normal distribution or as median/ lower-upper quartile range in the case of data with skewed or nonnormal distribution. Distribution of variables was evaluated by the Shapiro-Wilk test, and the quantilequantile plot. The homogeneity of variances was assessed by the Levene test. The two-way parametric ANOVA with post hoc contrast analysis, nonparametric Kruskal-Wallis test, or Mann-Whitney $U$ test were used in order to compare the data. In the case of skewed data distribution, logarithmic transformation was done before analysis.

\section{Results}

The IT procedure increased significantly the TAC amount in comparison to the sham $(p<0.001)$ and control groups $(p<0.01)$. The lowest level of this parameter was observed in the sham-operated group (control vs. sham $p<0.01$; Figure 2, Table 1). Also, the TOS parameter was significantly reduced in sham-operated rats. The control and IT groups showed a significantly higher TOS amount assessed in the liver tissue than sham-operated animals (control vs. sham $p<0.1$, IT vs. sham $p<0.01$; Figure 3, Table 1).

Significantly higher GPx activity was observed in the groups submitted to the IT and sham types of procedure when compared to the control group (control vs. sham $p<$ 0.001 , IT vs. sham $p<0.001$; Figure 4 , Table 1 ). The significant differences in the GR activity were found between the control, IT, and sham groups (control vs. sham $p<0.01$, IT vs. control $p<0.05$, IT vs. sham $p<0.001$; Figure 5, Table 1). The GR activity was significantly higher in control and IT-operated animals when compared to animals submitted to the sham procedure. The IT procedure significantly influenced CAT activity in comparison to control and sham-operated animals (IT vs. control $p<0.001$, IT vs. sham $p<0.001)$. After IT surgery, the CAT activity was twofold higher than in other studied animals (Figure 6, Table 1). Taking into consideration GST activity, there were no significant differences between the analysed groups observed (Table 1). The sham surgical intervention significantly increased total SOD activity when compared to control and IT-operated animals (control vs. sham $p<0.05$, IT vs. sham $p<0.05$; Figure 7, Table 1). A similar pattern for MnSOD activity was observed, where the highest activity of this enzyme was detected in sham-operated animals in comparison to the 


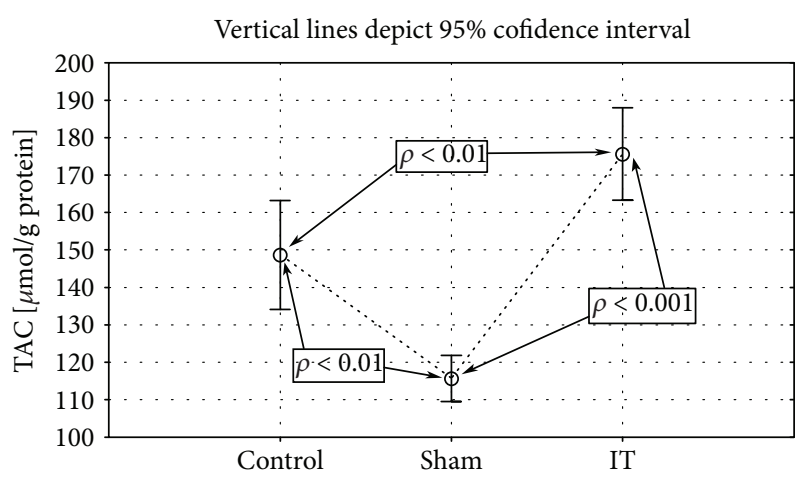

Figure 2: Mean values of TAC concentration in liver tissue of rats subjected to IT, sham operation type, and control group. Statistical significance was set at $p<0.001$. The statistical significance IT, sham operation type vs. control group was shown, and individual points are connected for the reader's convenience.

control and IT groups (control vs. sham $p<0.05$, IT vs. sham $p<0.001$; Figure 8, Table 1). CuZnSOD showed the same level of activity and no significant differences in the analysed groups: control, sham, and IT (Table 1).

\section{Discussion}

Bariatric surgery is currently one of the leading treatment options for morbid obesity, giving efficient and long-lasting results in reference to weight loss and glycemic control [16-18]. In this study, we have concentrated on the analysis of the influence of IT and sham procedures on the oxidative stress parameters measured in the liver tissue of animals 3 months after the IT and sham procedures: (i) the ileal transposition significantly increased TAC in comparison to shamoperated animals and the control group; (ii) animals after IT showed higher levels of TOS than sham-operated rats, and at the baseline, the total amount of TOS was congruous in IT and control study groups; (iii) GPx activity was increased in the groups submitted to the Sham and IT surgery in relation to control; (iv) GR and CAT were significantly higher after IT in comparison to the control and sham procedures; (vi) all analysed isoforms of SOD showed a similar pattern of activity in analysed groups. Total SOD and MnSOD were significantly higher in sham-operated animals in comparison to the IT intervention and control groups.

Obesity, a chronic disease, is interconnected with the augmentation of a broad range of health problems, which may lead to morbidity and mortality [19]. Under pathophysiological conditions such as overweight, T2DM, cardiovascular disease, and atherogenic processes, OS is induced and, successively, is associated with a defective production of adipokines, which further the progress of the metabolic syndrome $[19,20]$. TAC is understood as the additive action of all the antioxidants present in the selected tissue; hence, it is an integrated factor rather than the ordinary sum of measurable antioxidants, strongly modified by OS [21-23]. According to Serafini and Del Rio [23], TAC should be understood as a "conception" rather than an analytical technique, an idea of total antioxidant efficiency reflecting the complex aspects of redox interactions. Plasma TAC values were observed to be decreased in patients with type 1 diabetes and type 2 diabetes in comparison to matched healthy controls [24-26]. Other human studies also reported higher levels of oxidative stress, despite excessive antioxidant capacity, in the plasma of patients with uncomplicated type 2 diabetes compared with healthy control subjects [27]. The TAC concentration was decreased under the condition of obesity, which was associated with systemic antioxidant defence and increased oxidative stress [28]. The significant differences in TAC between the IT, sham, and control liver samples in the present study suggest that reduced total ROS content in the IT group is more likely due to increased scavenging of free radicals and other toxic species. Our observed increase in TAC in IT animals compared to Sham and controls further fosters evidence proposing that reduced oxidative stress after an IT procedure may be the main contributor for ameliorating insulin resistance and the negative consequences of obesity conditions. The levels of different oxidant species may be analysed separately, but the measurements are demanding, costly, and require advanced techniques [29]. Since the analysis of various oxidant molecules independently is not practical and their oxidant effects are additive, the body's oxidant/antioxidant status can be ascertained by measuring the TOS [29]. Therefore, TAC and TOS are more precise markers of the oxidative and antioxidative status of individuals and TOS levels can be understood as a general indicator of oxidant molecules [29]. 3 months after a sham procedure, liver antioxidant systems seem to be able to neutralize only part of the ROS produced, which was observed in significantly reduced TOS in the sham group when compared to the IT and control groups. We suggest that stress connected with sham intervention, which is a kind of control procedure without therapeutic outcomes, was too high and the system did not restore TOS to levels observed in other control and IT groups. We believe that the IT procedure showed a beneficial effect on the TOS amount in comparison to sham but did not improve the TOS amount significantly as was visible for the TAC amount.

$\mathrm{H}_{2} \mathrm{O}_{2}$ is metabolised by CAT and GPx and in that way reduced to water and molecular oxygen. GPx reduces $\mathrm{H}_{2} \mathrm{O}_{2}$ and organic peroxides (ROO) while oxidising glutathione (GSH) [30]. Oxidised glutathione (GSSG) is subsequently reduced back to GSH by glutathione reductase (GR) in the presence of NADPH (or the corresponding alcohol (ROH)) and GSSG. The reduced form of GSH is a key intracellular, most abundant antioxidant, which conjugates with electrophiles and takes a part in the metabolism and detoxification of endogenous compounds as well as xenobiotics [30]. Changes in glutathione status have been broadly reported in oxidative stress-related disorders, but the present study is the first comprehensively investigating the enzymes of glutathione status such as GR and GPx in the liver tissue of diabetic and obese Zucker rats after IT and sham surgery. The special attention is focused on the changes in GPx activity, as the expression of the enzyme is proved to be increased under conditions of excessive ROS production [31]. The increased values of GPx in the liver tissue of the animals after 
TABle 1: Antioxidant concentration and activity in liver tissue 3 months after IT surgery. Statistical significance was set at $p<0.05$.

\begin{tabular}{|c|c|c|c|c|c|c|c|}
\hline & Control & Sham & IT & $p$ & $\begin{array}{c}p_{1} \\
\text { Control vs sham }\end{array}$ & $\begin{array}{l}p_{2} \\
\text { IT vs control }\end{array}$ & $\begin{array}{c}p_{3} \\
\text { IT vs sham }\end{array}$ \\
\hline TAC $(\mu \mathrm{mol} / \mathrm{g})$ & $148.71 \pm 15.71$ & $115.63 \pm 6.68$ & $175.62 \pm 17.29$ & $<0.001$ & $<0.01$ & $<0.01$ & $<0.001$ \\
\hline TOS $(\mu \mathrm{mol} / \mathrm{g})$ & $0.27 \pm 0.07$ & $0.15 \pm 0.03$ & $0.26 \pm 0.05$ & $<0.001$ & $<0.01$ & 0.939 & $<0.01$ \\
\hline GPx (IU/g) & $72.02 \pm 15.77$ & $190.70 \pm 31.32$ & $161.21 \pm 35.24$ & $<0.001$ & $<0.001$ & $<0.001$ & 0.177 \\
\hline GR (IU/g) & $64.09 \pm 27.74$ & $34.22 \pm 11.40$ & $89.36 \pm 19.50$ & $<0.001$ & $<0.01$ & $<0.05$ & $<0.001$ \\
\hline CAT (IU/g) & $338.38 \pm 68.20$ & $333.22 \pm 42.85$ & $676.43 \pm 160.44$ & $<0.001$ & 0.996 & $<0.001$ & $<0.001$ \\
\hline Total SOD (NU/mg) & $477.12 \pm 44.51$ & $579.82 \pm 69.38$ & $478.24 \pm 96.53$ & $<0.05$ & $<0.05$ & 0.999 & $<0.05$ \\
\hline MnSOD (NU/mg) & $91.09 \pm 20.43$ & $126.10 \pm 38.10$ & $65.49 \pm 11.60$ & $<0.001$ & $<0.05$ & 0.145 & $<0.001$ \\
\hline GST (IU/g) & $35.29 \pm 1.63$ & $40.31 \pm 4.84$ & $38.16 \pm 6.46$ & 0.179 & - & - & - \\
\hline CuZnSOD (NU/mg) & $386.27 \pm 47.40$ & $439.41 \pm 60.06$ & $368.75 \pm 71.10$ & 0.086 & - & - & - \\
\hline
\end{tabular}

Abbreviations: GPX: glutathione peroxidase; GR: glutathione reductase; CAT: catalase, SOD: total superoxide dismutase; MnSOD: Mn superoxide dismutase; ZnSOD: Zn superoxide dismutase; GST: glutathione S-transferase: TAC: total antioxidant capacity; TOS: total oxidative status.

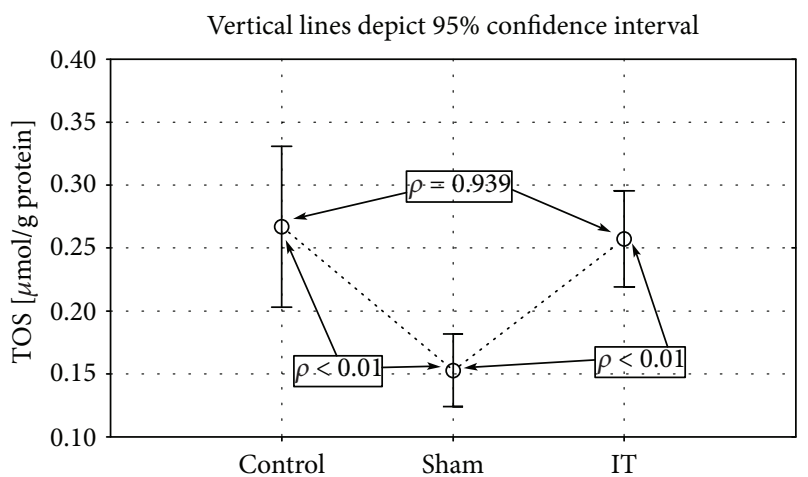

FIGURE 3: Mean values of TOS concentration in liver tissue of rats subjected to IT, sham operation type, and control group. Statistical significance was set at $p<0.05$. The statistical significance IT, sham operation type vs. control group was shown, and individual points are connected for the reader's convenience.

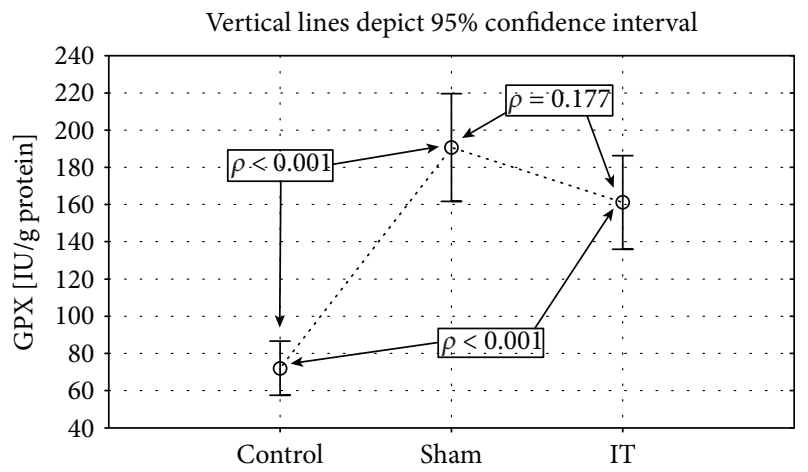

FIgUre 4: Mean values of GPx activity in liver tissue of rats subjected to IT, sham operation type, and control group. Statistical significance was set at $p<0.05$. The statistical significance IT, sham operation type vs. control group was shown, and individual points are connected for the reader's convenience.

the sham, but not IT procedure, may suggest that the disturbed liver glutathione status of rats might result from the enhancement of GSH consumption in the process catalysed

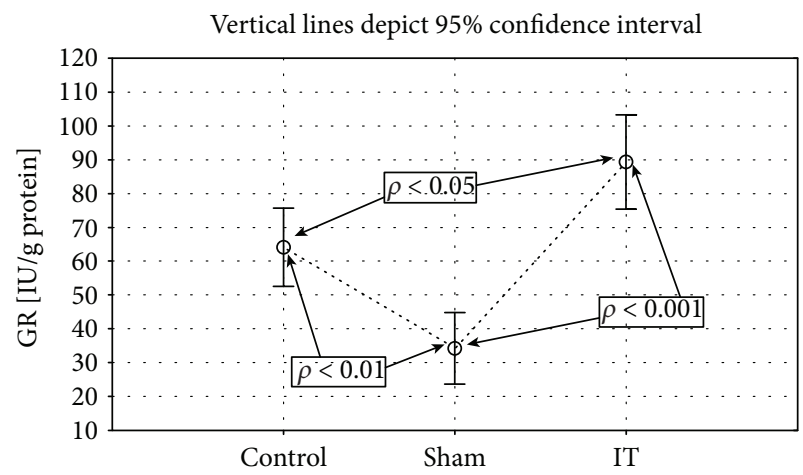

FIGURE 5: Mean values of GR activity in liver tissue of rats subjected to IT, sham operation type, and control group. Statistical significance was set at $p<0.05$. The statistical significance IT, sham operation type vs. control group was shown, and individual points are connected for the reader's convenience.

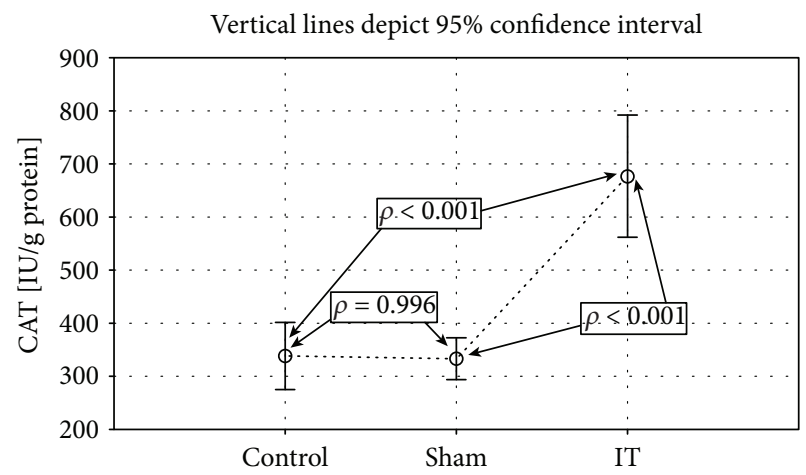

FIgURE 6: Mean values of CAT activity in liver tissue of rats subjected to IT, sham operation type, and control group. Statistical significance was set at $p<0.05$. The statistical significance IT, sham operation type vs. control group was shown, and individual points are connected for the reader's convenience.

by glutathione peroxidase. It appears feasible that the attenuation of oxidative stress accomplished by the IT treatment affects the expression of the GPx of glutathione metabolism, 


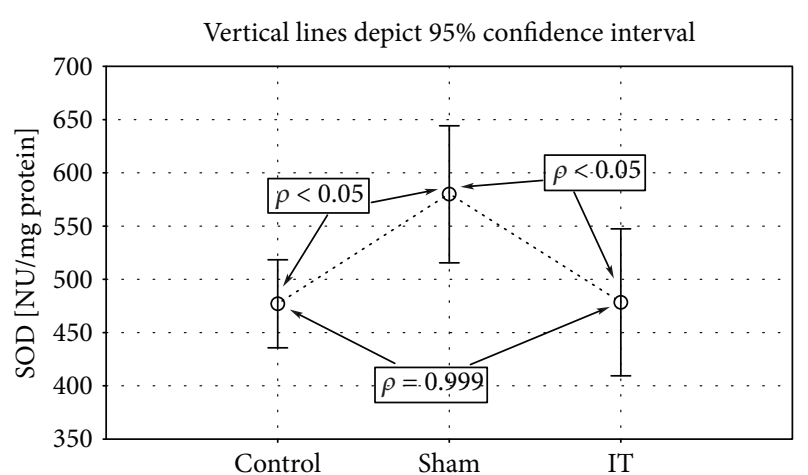

Figure 7: Mean values of Total SOD activity in liver tissue of rats subjected to IT, sham operation type, and control group. Statistical significance was set at $p<0.05$. The statistical significance IT, sham operation type vs. control group was shown, and individual points are connected for the reader's convenience.

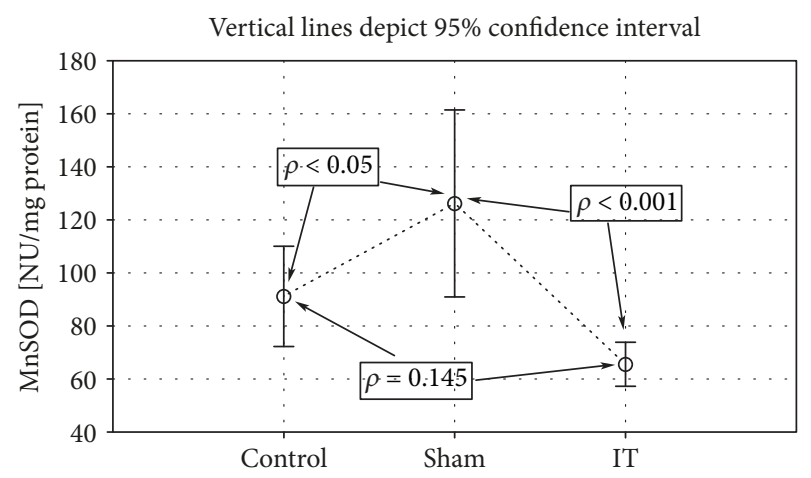

FIGURE 8: Mean values of MnSOD activity in liver tissue of rats subjected to IT, sham operation type, and control group. Statistical significance was set at $p<0.05$. The statistical significance IT, sham operation type vs. control group was shown, and individual points are connected for the reader's convenience.

which has been proposed to be ROS sensitive [31]. Other human studies proved the correlation between visceral fat accumulation and the enhanced oxidative status, where there is a significant decrease of GPx activity in the subgroups of the highest BMI category and the highest quartiles of waist circumference (WC) $[32,33]$. The positive, reductive impact of the IT procedure on the body weight of operated rats was already presented by Grüeneberger et al., and thus, we can assume that the antioxidant defence measured by GPx activity was significantly stronger in the IT but not in the sham group [7]. The control group, without any intervention, expressed the lowest level of GPx activity. The differences in the mobilization of the GPx/GR system in shamoperated animals may be understood as inefficiency or overloading of GR activity with the free radical formation when compared to the control and IT procedures. There was a significant increase in CAT enzymatic activity in IT-operated animals. This can be a positive effect of the bariatric procedure on the CAT antioxidative defence as it is known that CAT activity was found to be significantly diminished upon the increase of adipose tissue [19].
Under the conditions of long persisting obesity, the pool of antioxidant sources can be diminished, further affecting the activity of enzymes such as superoxide dismutase (SOD). In human subjects, the activity of SOD in obese individuals was significantly reduced in comparison to that in healthy subjects, intensifying the development of obesityrelated health problems [19]. Obesity has been connected with an enhanced expression of NADPH oxidase and a restriction in the expression of various antioxidant proteins. In this study, which is an animal model of obesity, the total SOD and MnSOD measured in the sham group were significantly increased in comparison to the IT and control groups. The elevation of SOD activity may be understood as amplification of antioxidant capacity in sham animals and consequently a reduction in oxidative lesions. It is essential to indicate that an increase in total SOD, MnSOD, and CAT activities after bariatric surgery can be related to the time after surgery and reduction of adipose tissue. It is known that metabolic surgery improves the inflammatory response during the medium term after surgery. Human studies have found that oxidative stress was significantly reduced 6 months after bariatric surgery $[19,34,35]$. Murri et al. reported that 9 months after biliopancreatic diversion, the TAC and the activity of CAT and SOD did not change significantly during the study [36]. It is suggested that a long-term effect of bariatric surgery observed by reduced mass of adipocytes, and thus decline in the synthesis of molecules directly related to hypertrophic adipose tissue, might balance the antioxidant response.

\section{Conclusions}

We conclude that the IT procedure had a positive impact on the reduction of oxidative stress, measured by TAC and TOS parameters in the liver tissue of obese rats. The dynamic, adaptive, and protective mechanisms of enzymatic antioxidant systems were observed after the IT but not sham procedure. Nevertheless, 3 months after surgery, the midterm effect of bariatric surgery was observed, which might not fully balance the antioxidative response.

\section{Data Availability}

The data used to support the findings of this study are available from the corresponding author upon request.

\section{Ethical Approval}

All applicable institutional and/or national guidelines for the care and use of animals were followed. All animal experimental protocols were approved by the Local Ethics Committee, Poland.

\section{Conflicts of Interest}

The authors declare that they have no conflict of interest. 


\section{Authors' Contributions}

BSP, DS, and KWK conceived the idea of the experiment; TS and JP maintained the animals; EC and DS performed the statistical analysis of the data; BSP and ER carried out overall analysis; and DS, BSP, AD, and WK wrote the manuscript. All authors have approved the final version of the manuscript. Work was performed in cooperation between Ludwig Maximilian University of Munich, Germany, and the University of Silesia in Katowice, Poland.

\section{Acknowledgments}

The authors would like to thank Anna Dulska for illustration and graphic design. Authors would like to express their special gratitude to Mr. Scott Richards for scientific English language correction. This work was funded by the Medical University of Silesia, Poland.

\section{References}

[1] C. Patel, H. Ghanim, S. Ravishankar et al., "Prolonged reactive oxygen species generation and nuclear factor-kappaB activation after a high-fat, high-carbohydrate meal in the obese," The Journal of Clinical Endocrinology and Metabolism, vol. 92, no. 11, pp. 4476-4479, 2007.

[2] SOReg, "Yearly report SOReg 2012. Weight changes, comorbidity and long-term complications," 2016, http://www.ucr.uu.se/ soreg.

[3] J. Sundström, G. Bruze, J. Ottosson, C. Marcus, I. Näslund, and M. Neovius, "Weight loss and heart failure: a nationwide study of gastric bypass surgery versus intensive lifestyle treatment," Circulation, vol. 135, no. 17, pp. 1577-1585, 2017.

[4] X. J. Xu, C. Apovian, D. Hess, B. Carmine, A. Saha, and N. Ruderman, "Improved insulin sensitivity 3 months after RYGB surgery is associated with increased subcutaneous adipose tissue AMPK activity and decreased oxidative stress," Diabetes, vol. 64, no. 9, pp. 3155-3159, 2015.

[5] C. K. Furuya Jr, C. P. M. S. de Oliveira, E. S. de Mello et al., "Effects of bariatric surgery on nonalcoholic fatty liver disease: preliminary findings after 2 years," Journal of Gastroenterology and Hepatology, vol. 22, no. 4, pp. 510-514, 2007.

[6] H. S. Koopmans, A. Sclafani, C. Fichtner, and P. F. Aravich, "The effects of ileal transposition on food intake and body weight loss in vmhobese rats," The American Journal of Clinical Nutrition, vol. 35, no. 2, pp. 284-293, 1982.

[7] J. M. Grüeneberger, T. Fritz, C. Zhou et al., "Long segment ileal transposition leads to early amelioration of glucose control in the diabetic obese Zucker rat," Videosurgery and Other Miniinvasive Techniques, vol. 2, pp. 130-138, 2013.

[8] S. Nakanishi, T. Kuramoto, N. Kashiwazaki, and N. Yokoi, "Genetic profiling of two phenotypically distinct outbred rats derived from a colony of the Zucker fatty rats maintained at Tokyo Medical University," Experimental Animals, vol. 66, no. 2, pp. 91-98, 2017.

[9] O. Erel, "A novel automated direct measurement method for total antioxidant capacity using a new generation, more stable ABTS radical cation," Clinical Biochemistry, vol. 37, no. 4, pp. 277-285, 2004.

[10] I. Carlberg and B. Mannervik, "Glutathione reductase," Methods in Enzymology, vol. 113, pp. 484-490, 1985.
[11] H. Aebi, "Catalase in vitro," Methods in Enzymology, vol. 105, pp. 121-126, 1984.

[12] B. Mannervik, "Glutathione peroxidase," Methods in Enzymology, vol. 113, pp. 490-495, 1985.

[13] W. H. Habig and W. B. Jakoby, "Assays for differentiation of glutathione S-transferases," Methods in Enzymology, vol. 77, pp. 398-405, 1981.

[14] Y. Oyanagui, "Reevaluation of assay methods and establishment of kit for superoxide dismutase activity," Analytical Biochemistry, vol. 142, no. 2, pp. 290-296, 1984.

[15] O. H. Lowry, N. Rosebrough, A. Farr, and R. Randall, "Protein measurement with the Folin phenol reagent," The Journal of Biological Chemistry, vol. 193, no. 1, pp. 265-275, 1951.

[16] S. A. Brethauer, H. M. Heneghan, S. Eldar et al., "Early effects of gastric bypass on endothelial function, inflammation, and cardiovascular risk in obese patients," Surgical Endoscopy, vol. 25, no. 8, pp. 2650-2659, 2011.

[17] G. Mingrone, S. Panunzi, A. de Gaetano et al., "Bariatric surgery versus conventional medical therapy for type 2 diabetes," The New England Journal of Medicine, vol. 366, no. 17, pp. 1577-1585, 2012.

[18] P. R. Schauer, D. L. Bhatt, J. P. Kirwan et al., "Bariatric surgery versus intensive medical therapy for diabetes - 5-year outcomes," The New England Journal of Medicine, vol. 376, no. 7, pp. 641-651, 2017.

[19] A. Fernández-Sánchez, E. Madrigal-Santillán, M. Bautista et al., "Inflammation, oxidative stress, and obesity," International Journal of Molecular Sciences, vol. 12, no. 5, pp. 31173132, 2011.

[20] K. Esposito, M. Ciotola, B. Schisano et al., "Oxidative stress in the metabolic syndrome," Journal of Endocrinological Investigation, vol. 29, no. 9, pp. 791-795, 2006.

[21] C. Chrysohoou, D. B. Panagiotakos, C. Pitsavos et al., "The implication of obesity on total antioxidant capacity in apparently healthy men and women: the ATTICA study," Nutrition, Metabolism \& Cardiovascular Diseases, vol. 17, no. 8, pp. 590597, 2007.

[22] G. Bartosz, "Total antioxidant capacity," Advances in Clinical Chemistry, vol. 37, pp. 219-292, 2003.

[23] M. Serafini and D. Del Rio, "Understanding the association between dietary antioxidants, redox status and disease: is the total antioxidant capacity the right tool?," Redox Report, vol. 9, no. 3, pp. 145-152, 2004.

[24] J. Valabhji, A. J. McColl, W. Richmond, M. Schachter, M. B. Rubens, and R. S. Elkeles, "Total antioxidant status and coronary artery calcification in type 1 diabetes," Diabetes Care, vol. 24, no. 9, pp. 1608-1613, 2001.

[25] E. C. Opara, E. Abdel-Rahman, S. Soliman et al., "Depletion of total antioxidant capacity in type 2 diabetes," Metabolism, vol. 48, no. 11, pp. 1414-1417, 1999.

[26] A. Ceriello, N. Bortolotti, E. Falleti et al., "Total radicaltrapping antioxidant parameter in NIDDM patients," Diabetes Care, vol. 20, no. 2, pp. 194-197, 1997.

[27] O. Savu, C. Ionescu-Tirgoviste, V. Atanasiu, L. Gaman, R. Papacocea, and I. Stoian, "Increase in total antioxidant capacity of plasma despite high levels of oxidative stress in uncomplicated type 2 diabetes mellitus," The Journal of International Medical Research, vol. 40, no. 2, pp. 709-716, 2012.

[28] S. H. Lim, S. H. Fan, and Y. H. Say, "Plasma total antioxidant capacity (TAC) in obese Malaysian subjects," Malaysian Journal of Nutrition, vol. 18, no. 3, pp. 345-354, 2012. 
[29] O. Erel, "A new automated colorimetric method for measuring total oxidant status," Clinical Biochemistry, vol. 38, no. 12, pp. 1103-1111, 2005.

[30] H. Zhang and H. J. Forman, "Glutathione synthesis and its role in redox signaling," Seminars in Cell \& Developmental Biology, vol. 23, no. 7, pp. 722-728, 2012.

[31] E. Lubos, J. Loscalzo, and D. E. Handy, "Glutathione peroxidase-1 in health and disease: from molecular mechanisms to therapeutic opportunities," Antioxidants \& Redox Signaling, vol. 15, no. 7, pp. 1957-1997, 2011.

[32] S. Araki, K. Dobashi, Y. Yamamoto, K. Asayama, and $\mathrm{K}$. Kusuhara, "Increased plasma isoprostane is associated with visceral fat, high molecular weight adiponectin, and metabolic complications in obese children," European Journal of Pediatrics, vol. 169, no. 8, pp. 965-970, 2010.

[33] P. Matusik, Z. Prokopowicz, B. Norek, M. OlszaneckaGlinianowicz, J. Chudek, and E. Malecka-Tendera, "Oxidative/antioxidative status in obese and sport trained children: a comparative study," BioMed Research International, vol. 2015, Article ID 315747, 8 pages, 2015.

[34] T. Sledzinski, E. Goyke, R. T. Smolenski, Z. Sledzinski, and J. Swierczynski, "Decrease in serum protein carbonyl groups concentration and maintained hyperhomocysteinemia in patients undergoing bariatric surgery," Obesity Surgery, vol. 19, no. 3, pp. 321-326, 2009.

[35] N. Gletsu-Miller, J. M. Hansen, D. P. Jones et al., "Loss of total and visceral adipose tissue mass predicts decreases in oxidative stress after weight-loss surgery," Obesity, vol. 17, no. 3, pp. 439-446, 2009.

[36] M. Murri, E. García-Fuentes, J. M. García-Almeida et al., "Changes in oxidative stress and insulin resistance in morbidly obese patients after bariatric surgery," Obesity Surgery, vol. 20, no. 3, pp. 363-368, 2010. 


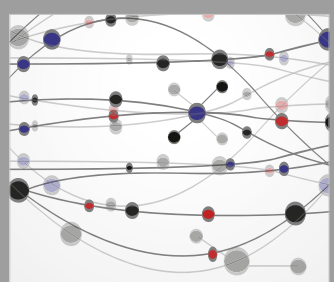

The Scientific World Journal
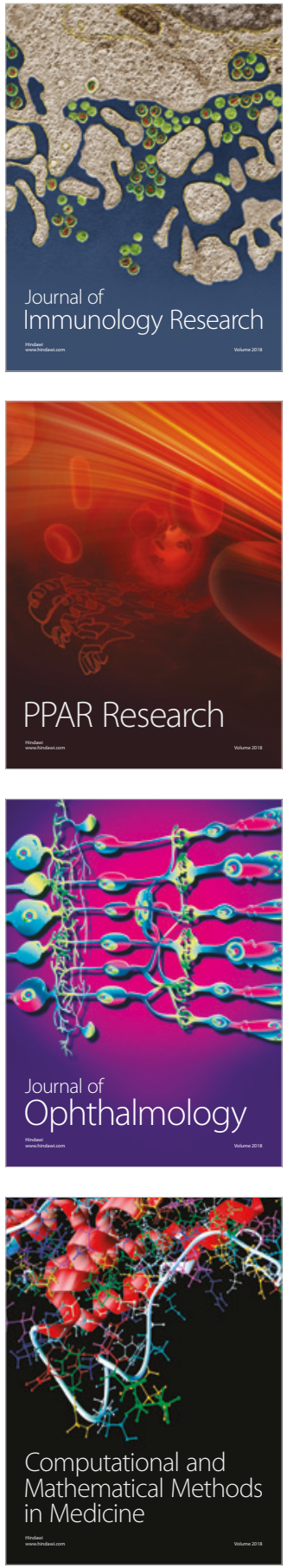

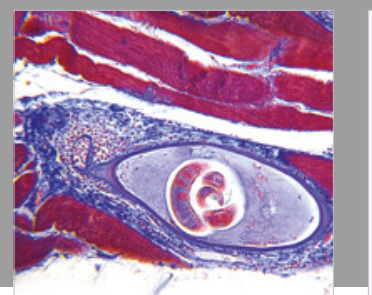

Gastroenterology Research and Practice

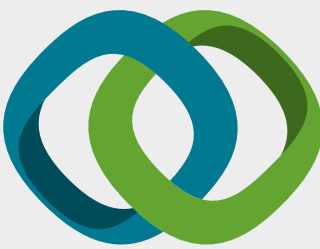

\section{Hindawi}

Submit your manuscripts at

www.hindawi.com
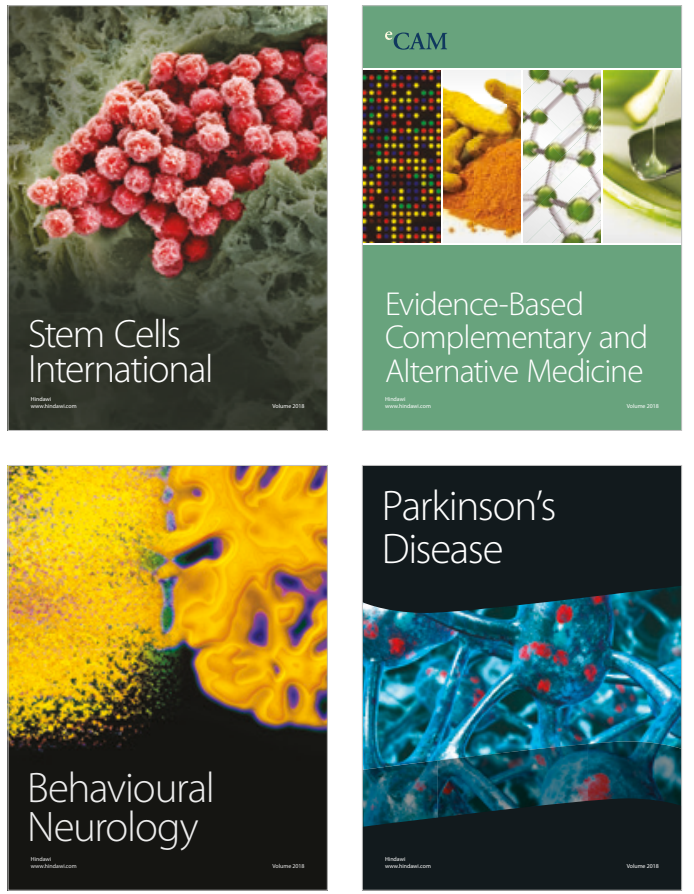

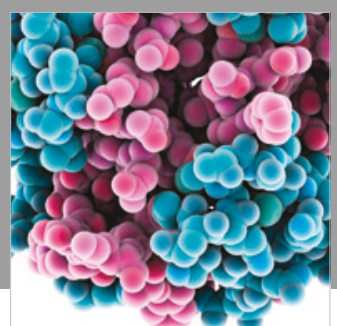

ournal of

Diabetes Research

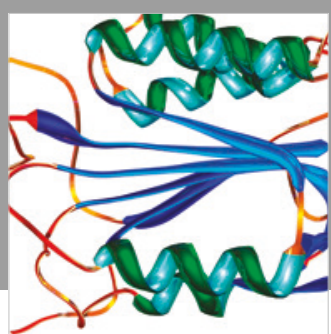

Disease Markers
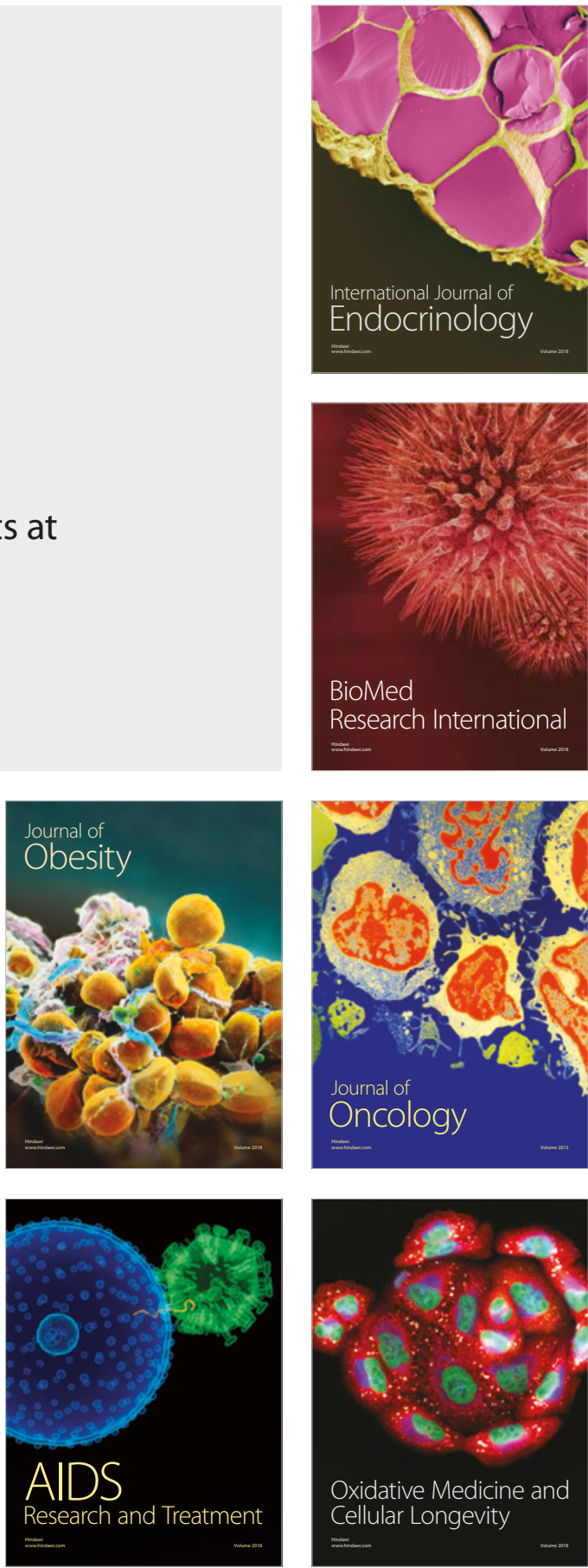